(C)2009 IEEE. Personal use of this material is permitted. However, permission to reprint/republish this material for advertising or promotional purposes or for creating new collective works for resale or redistribution to servers or lists, or to reuse any copyrighted component of this work in other works must be obtained from the IEEE. 


\section{Processing of Low Level Signals in Mixed Signal Environment -A Hardware Design for Better Performance}

\author{
Atif Sharif, Vidyasagar. M. Potdar, Jaipal Singh \\ Digital Ecosystems and Business Intelligence Institute \\ Curtin University of Technology \\ Perth, Australia \\ atif.sharif@postgrad.curtin.edu.au,\{v.potdar,j.singh\}@curtin.edu.au
}

\begin{abstract}
The Low level signals faces many problems during their processing and digitization. The main affecting factor is the added noise during its propagation in mixed signal environment which makes very difficult to extract exact information from the signal. Additionally hardware requirement increases when we want to process these kinds of signals. In this paper, we proposed a customized hardware for this purpose. This hardware caters the problems of noise, signal integrity issues and crosstalk which directly effects embedded circuit efficiency. The board has all the options to cater the situation where the required signal is buried in the noise or having very low level amplitude. Noise elimination and crosstalk is avoided in this hardware which include high speed Field Programmable gate Array (FPGA).
\end{abstract}

Index Terms - crosstalk; signal integrity; PCB; $A D C ; D A C$; FPGA's.

\section{INTRODUCTION}

The signals exist in this world are analog in nature. For example audio or voice signal, temperature etc. In most cases we have to process the analog signals. In past, the processing was done in analog domain using analog electronics. Now with availability of high speed digital signal processors and FPGA's most of the signal processing will be done in digital domain. Normal analog signals, which are strong in magnitude, are directly fed to the input of the Analog-to-Digital Converters (ADCs) without any concern.

However, when the analog signal is in microvolt or very small in magnitude then the problem comes how to handle this kind of signal especially when the signal is buried in noise. When we have to process such signals, the problem comes in terms of noise addition, crosstalk and amplification without noise. The signal processing boards that are readily available in the market mostly have the digital section on it that incorporates components like

\author{
*Rana Fayyaz Ahmad, ${ }^{* *}$ Malaya Nath, \\ ${ }^{* * *}$ Yan Yang \\ *Electrical Engineering Department \\ ${ }^{*}$ NUST, ${ }^{* *}$ IIT, ***Seikei University \\ *Pakistan, ${ }^{* *}$ India, ${ }^{* * *}$ Japan, \\ "fayyaz@aero.com.pk, "*malaya.nath@gmail.com, \\ *** yan-yang@ejs.seikei.ac.jp
}

FPGA, ADCs and Digital-to-Analog Converters (DACs). Direct input for analog signals of the form audio or video are also present on these boards. These boards are good for their use in digital signal Processing applications or for processing analog signals having larger magnitude. Integration of low level analog signals with them makes a job bit difficult in two ways. Firstly we need a separate card designed for these low level signals whose purpose is to provide the desired amplification level without noise so that it can be directly fed to the input of these processing boards. However, this will not produce good results, as we are integrating two separate hardwares. Secondly for low level signals we need a board which has the capability to avoid the noise mix-up in the analog section from the digital section. With this the effect of crosstalk would not be present on the board. So a need arises for customized hardware to cater the above mentioned problems, which in-turn be solved only with designing of good hardware for better performance.

The rest of the paper is organized as fallows in section Problem Definition (Section II) we are giving an in-depth overview of the actual problem which normally be evident in the real world. Section III describes the proposed board design of the customized hardware platform for signal processing of noisy low-level signals followed by the Experimental setup and results (Section IV) and then finally the evaluation is discussed in Section V.

\section{PROBLEM DEFINITION}

The low level signals are of different types. Some are generated from transducers, audio and optical signals which are converted into the electrical form. To extract information from these kinds of signals it is desired that they should have less added noise at the receiver side or at processing input. The main problem is the added noise which is picked up by these signals when they travel along the PCB. Before 
digitization the analog signal should be properly cleaned and noise free. The signal level should be at least more than half Least Significant Bit (LSB) of the ADC in order to retrieve the correct information. It is therefore desired that the signal should be cleaned from noise in analog section and also amplified to such a level that it can be easily processed in digital domain. This happens only when analog signal is properly routed on the PCB and care should be taken in power supply which is given to the circuit [2]. The power supply noise should be removed so that it does not mix with the low level analog signals.

The following areas should be addressed when we have to process the low level analog signals

- Separation of analog and digital signals

- Concept of Split planes

- Grounding Issues

- Power Supply filtering

\section{A. Separation of analog and digital signals}

Analog and digital signals are not to be used together on the same board. It should be separated from each other in terms of grounding and power supply. The routing of the analog signal should be carried out in such a way that it should not pass through the digital area. It is clearly a good practice to separate the Analog and Digital portions of the Circuit. Clearly mark the Analog and Digital signals [1]. Similarly clearly mention the analog and digital power supply in the circuit. This will help in designing of the PCB.

\section{B. Concept of Split planes}

The concept of Split planes in the PCB is very effective in a sense that it eliminates the free mixing of the analog signals with high speed digital signals [3]. By doing this, the analog signals remain confined in the analog portion. The current return loop only passes through the analog region and do not pick noise from the digital region. Similarly, High speed signals remain in their area of concern and do not mix with the low level analog signals. These ground and power planes in multilayer design are also splitted into the analog and the digital portions so that they provide power and ground to the analog and digital areas separately.

\section{Grounding Issues}

The ground provides the return path to the signals. If we mix analog and digital grounds together then the noise from the digital signals through ground will be coupled into the low level analog signals. This degrades the performance of the system and the output which we require from the analog signal will be diminished in the noise.

As we marked the digital and analog grounds in the circuit, every split plane has its own ground, for example when we nominate some area on the board as analog so we provide analog ground to that area so that return path should be from analog ground and vice versa.

\section{Power Supply Filtering}

Power supply plays an important role in noise addition especially when a single supply is used to run a circuit which has both analog and digital sections, so it contains all the noise present in the circuit. This noise degrades the quality of analog signals and can be eliminated by doing the efficient filtering in the circuit [6]. The following areas needs careful attention

- Pre-Filtering at Input Supply

- Separate filtering for power Supply inside the board

- $\quad$ Special Filtering at Low Noise Analog

1) Pre-Filtering at Input Supply:

It is not recommended to use the direct supply to the circuit. The best approach is to filter at the input connector which is on the board. Use some basic filtering techniques like simple RC or LC filtering to eliminate the power supply noise.

\section{2) Separate filtering for power Supply inside the} board:

Generally the clean power supply should be routed to the analog and digital areas. So for more noise rejection, we use special Pi Filtering Network at the input of the analog section. Use of this kind of filter, which is simple LC Pi Network, will eliminate the noise present in the supply. By doing this more clean supply is fed to the circuit.

\section{3) Special Filtering at Low Noise Analog section:}

The analog supply is filtered at its input section before feeding to different analog ICs like operation amplifiers, Transimpedance amplifier and ADCs. If an analog section has a circuit like Transimpedance amplifier or some input stage which pre-process and amplify the low level analog signals whose value is in microvolts then a care should be taken to provide more clean supply to the circuit. For this we can make use of 
simple RC network to filter our analog supply. This will eliminate the noise more effectively and consequently suppresses noise during the signal amplification.

\section{PROPOSED BOARD DESIGN}

In this paper we propose a better design of the circuit as well as PCB which is based on those considerations mentioned above. Our input analog signal is of low amplitude i.e. few microvolts and is of two types

a) Audio signal/Speech Signal

b) A signal from photodiode

The requirement is to process these kinds of signals in analog and digital domains. We used Xilinx FPGA, High Speed ADC and DACs in our design. For Analog section, we used high speed as well as low noise operational amplifiers. These amplifiers are used to amplify low level signals into milivolt or volts depending upon the gain which we will set. So the combination of two sections i.e. analog and digital demands a very efficient board level designing (mixed signal design). The board design is divided into two stages

$$
\begin{array}{ll}
1 . & \text { Circuit Design. } \\
2 . & \text { PCB Design. }
\end{array}
$$

\section{Circuit Design}

In circuit design we have taken care of all the aspects mentioned above in Section II of the paper. The filtering in the design is carried out very efficiently. LC and RC filtering in analog sections, depending upon the circuit design requirements, are used. The total supply filtering is shown in Fig 1.

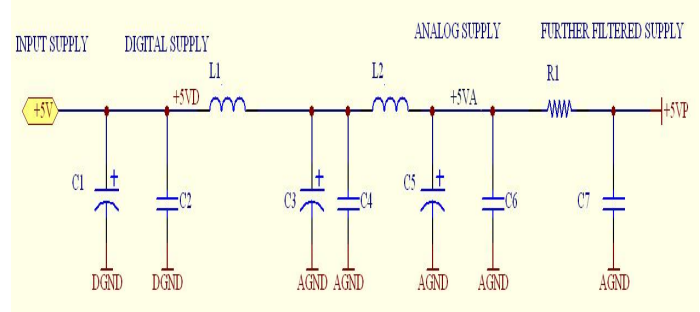

Fig. 1. Power stage: Filtering stage

Different parts of the supply will be used in different design sections. The input to the circuit is $+5 \mathrm{~V}$ and initial filtering is carried out by $\mathrm{C} 1$ and $\mathrm{C} 2$. These two capacitors provide initial power supply filtering. This supply goes further towards digital section. The analog supply is taken from the output of L2 i.e. +5VA after additionally performing the LC filtering in analog section. At the end the input stage is derived from the output of $\mathrm{RC}$ filtering i.e. $+5 \mathrm{VP}$.

This kind of filtering solves the problem of noisy supply. Similarly, proper grounding the analog and digital signals to their respective grounds is also of key importance in the circuit design. These grounds/references are marked with Analog ground (AGND) and Digital ground (DGND) and will be placed in their respective areas (Analog and Digital). We separated our circuit in three different sections as shown in Fig 2.

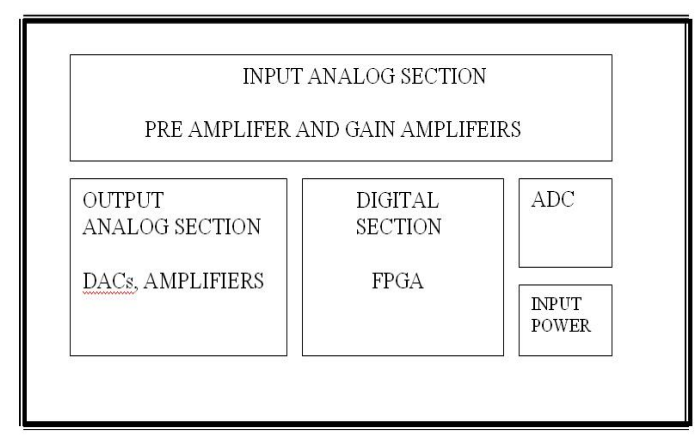

Fig. 2. Proposed circuit blocks

The above figure shows the division made in such a way that analog and digital areas are located separately. This layout will provide the base for the PCB design. Based on this separation we can easily target our design for high performance.

\section{PCB Design}

The design of PCB for the processing of low level signals is based on the above separation shown in Fig. 2. The PCB design is a very important and critical stage in handling and processing the low level signals in mixed signal environment.

The following decisions are made in designing the PCB.

\subsection{PCB layer stack up}

PCB stack-up is an important factor in determining the EMC performance of a board. A good stack-up can be very effective in reducing radiation from the loops on the PCB [3].

Four factors are important with respect to board stack-up considerations: 
- The number of layers

- The number and types of planes (power and ground) used

- The ordering or sequence of the layers

- The spacing between the layers

Multi-layer boards using ground and power planes provide significant reduction in radiated emission noise over two layer PCBs [3]. The six layer PCB design strategy is suitable to handle the task. In multilayer design, stack up of the layers plays an important role in performance of the board. For example a signal layer should be adjacent to the ground plane and it should be tightly coupled to the plane. This will provide better current return paths to the signal routed on the top or bottom layers. Also ground and power planes are placed closely together so that it can provide a better decoupling. The two planes then form a large decoupling capacitive effect. High speed signals should be properly routed in the internal layers so that it will not effect the other signals. Multiple ground planes reduce the ground impedance so it should be recommended. The ordering sequence has an effect on the performance of the PCB, we cannot place layers in any order, and it is therefore recommended that the ground layer should be placed between the signal layer and power plane. The overall benefit would be twofold. Firstly, it provides a close return path to the signals present on the signal layer and secondly it tightly couples the $+5 \mathrm{~V}$ power plane with the ground plane. The spacing between the layers should be as least as possible. Mostly, between the ground and power plane which is better to form good decoupling effect. Based on these factors we have chosen the PCB layer stack up in this manner as shown below Fig 3.

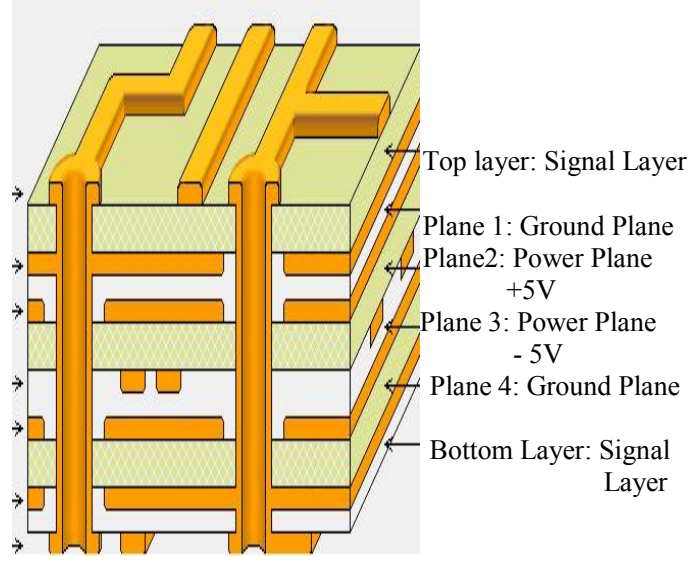

Fig.3. Various layers for the $\mathrm{PCB}$
This kind of stack up provides very good results. Both signal layers are tightly coupled with ground planes. Also both power planes are close to their respective grounds. This design approach has enhanced the PCB performance in terms of noise rejection and crosstalk.

\subsection{Split Planes}

In mix signal envoirment, the concept of split planes plays an important role. It does not allow the free mixing of the analog and digital signals. The Planes are splitted into analog and digital in a way according to the circuit and type of signal. We split the board in according to our circuit layout shown in Fig. 2. The internal view of the split planes is shown in Fig.4

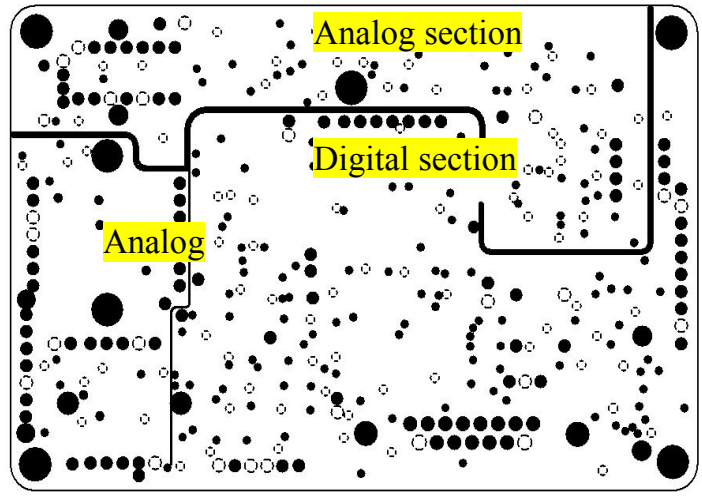

Fig.4. Various planes in the proposed board

Fig.4 shows the three different areas marked analog and digital. The connection of the ground planes are under the ADCs. Because ADC is placed in such a way that the one half of its region in analog plane and half in digital plane. The manufactured board is shown below in Fig. 5

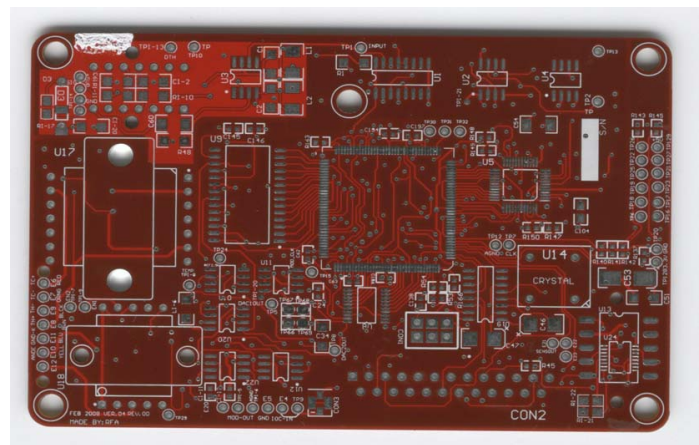

Fig.5. Designed PCB for Signal Processing board 


\section{EXPERIMENTAL SETUP AND RESULTS}

The proposed PCB was stuffed with components like FPGA, ADC and analog amplifiers and other passive elements etc. To test the board the instruments like HP spectrum/Network Analyzer 3589 (10 Hz-150 MHz), Power supply, Digital storage Scope etc were used. FPGA derived from $10 \mathrm{MHz}$ crystal Clock and it is further divided in FPGA to $1 \mathrm{MHz}$ for ADC sampling. The clock present in digital section is monitored by HP spectrum analyzer and shown below in Fig.6. It contained all the harmonics present in it and as well as noise present in digital region.

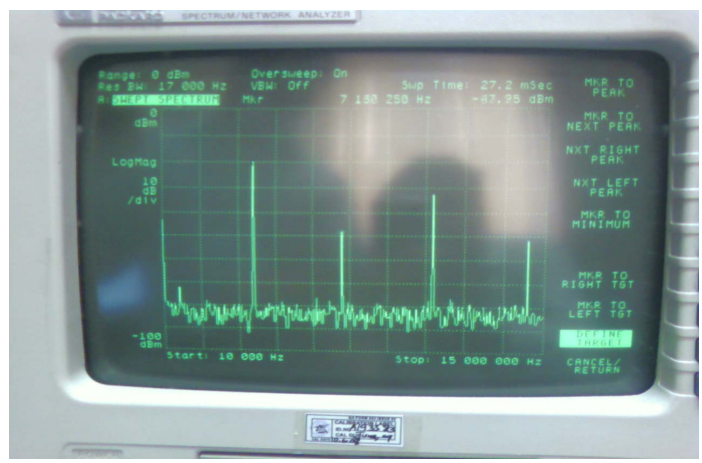

Fig. 6. Various harmonics and noise in the Digital plane

Now we measure the crosstalk and noise level coupled in the analog section. First, there was no input applied to the analog section. We then monitored the input of the system for any possible frequency component from the digital section comes to affect the low level signals. The result is shown in Fig. 7. The figure shows no crosstalk was observed in the input analog section and also no harmonic was found present in the analog section. The noise floor level was in between -90 to $-100 \mathrm{dbm}$ so it will not affect our low level analog signals which are in the range of microvolts.

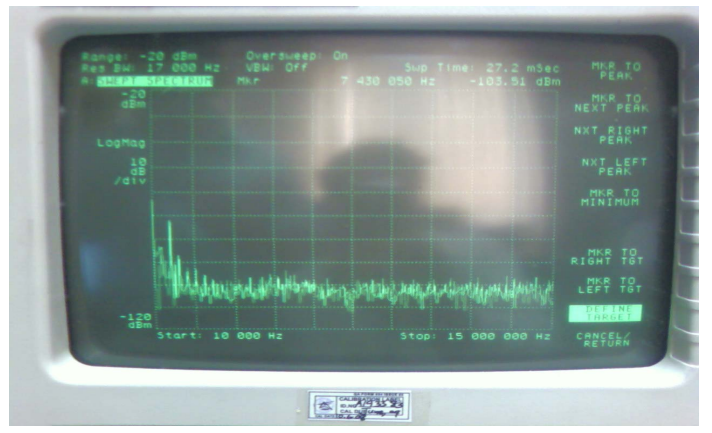

Fig.7. Snapshot of noise floor taken by spectrum analyzer

\section{EVALUATION}

The proposed board design shows better result as compared to the simple digital or double sided boards used for the signal processing of the low level analog signals. The Multilayer Design, with effective use of the design rules, we can successfully process the low level analog signals up to the desired limitations. Our hardware is based on all the concepts presented in this paper. The signal obtained after amplification had the less noise. The further processing of the signal was carried out in the FPGA. The output from the FPGA again fed into the analog section for possible feedback scenario. These Results are compared with the previously designed double sided board used for the signal processing shown in Fig. 8

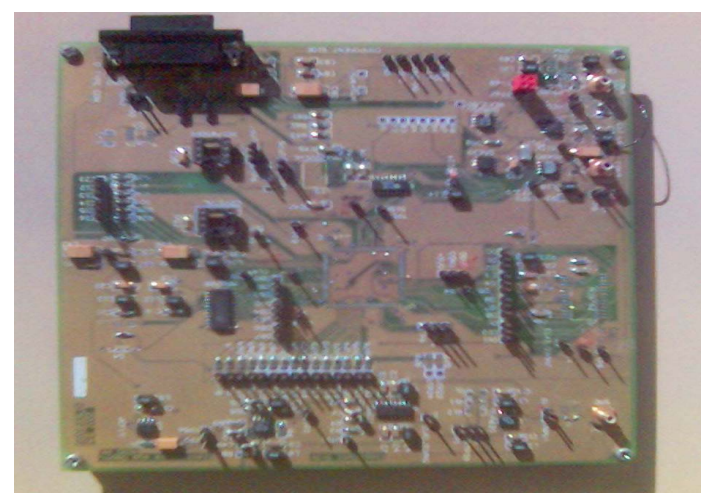

Fig. 8. Double layer card

However, for possible improvement in double sided design

- Ground plane should be added above and below i.e. copper pouring on top side and bottom side of the PCB. This reduces the ground resistance and crosstalk effects.

- The analog plane is also splitted from the digital plane.

However, this board did not performed well for the low level signals due to its less noise rejection capability. The multilayer design solves the problem based on the design guidelines provided in this paper.

We only compared two major factors; one is crosstalk level generated at the analog input of the board and second is the noise level present at the input side. 
TABLE 1 COMPARISON BETWEEN TWO BOARDS

\begin{tabular}{|l|l|l|}
\hline $\begin{array}{l}\text { Number of } \\
\text { Layers }\end{array}$ & Crosstalk Level & $\begin{array}{l}\text { Noise } \\
\text { Level }\end{array}$ \\
\hline $\begin{array}{l}\text { Double } \\
\text { Sided PCB }\end{array}$ & $\begin{array}{l}-40 \mathrm{dbm} \text { (Fundamental } \\
\text { frequency component from } \\
\text { FPGA clock) }\end{array}$ & $-60 \mathrm{dbm}$ \\
\hline $\begin{array}{l}\text { 6-Layer PCB } \\
\text { Design }\end{array}$ & $\begin{array}{l}\text { No Harmonic components } \\
\text { are found. }\end{array}$ & $-100 \mathrm{dbm}$ \\
\hline
\end{tabular}

From the table it is evident that the crosstalk is suppressed in six layer design. Also in double layer card design the fundamental harmonic is present on the board at level of $-40 \mathrm{dbm}$ (as seen from spectrum analyzer measurements) whereas the noise level in the six layer design is decreased up to $-100 \mathrm{dbm}$ which is quite significant.

\section{REFERENCES}

[1] Eric Bogatin, "Signal Integrity-Simplified". Modern Semiconductor Design series: Prentice Hall-2004.

[2] Martin O' Hara, "EMC at PCB and Component Level" Newness-1998.

[3] Ott, H. W., Partitioning and Layout of a Mixed Signal PCB, Printed Circuit Design, June 2001.

[4] Walter C Bosshart, "Printed Circuit Board-Design and Technology" McGraw HILL -1994

[5] Johnson,H.W., and Gram,M. "High Speed Digital Design". Prentice Hall, 1993.

[6] Filtering Techniques: Isolating analog and digital Power supplies, Application report, Texas Instruments 2001. 